\title{
A STUDY OF ANTIFIBRINOLYSIN ACTIVITY IN THE PLASMAS OF VARIOUS ANIMAL SPECIES ${ }^{1}$
}

\author{
BY M. MASON GUEST, BYRNE M. DALY, ARNOLD G. WARE, \\ AND WALTER H. SEEGERS \\ (From the Department of Physiology, Wayne University College of Medicine, \\ Detroit, Michigan)
}

(Received for publication June 1, 1948)

This report describes methods which have been developed in this laboratory for the determination of antifibrinolysin activity in plasma. Using these methods, studies have been made of the antifibrinolysin activity in the plasma of the alligator (Alligator mississippiensis), guinea pig (genus Cavia), cow (Bos taurus), rat (Rattus norvegicus, Sprague-Dawley strain), frog (Rana pipiens), man (white, male), cat (Felis domestica), dog (Canis familiaris, mongrel breeds), rabbit (genus Lepus, mixed strains), opossum (Didelphis virginiana), chicken (genus Gallus, Plymouth Rock), and pigeon (Columbia livia). With the exception of man, both sexes were represented but pregnant animals were not utilized in the sampling. Graphs are presented which indicate the relationships between plasma concentration (antifibrinolysin units) and the time required for the clots to dissolve. With the help of these graphs it is possible to measure the unit antifibrinolysin activity in a plasma sample from any of the species which have been studied.

The terminology used in this report will be the one recently suggested by Loomis, George and Ryder (1) and by Astrup and Permin (2). Considerable confusion has existed in the past regarding nomenclature and we believe that a uniform terminology will assist greatly in the development of this field.

Antifibrinolysin (Fibrinolysin Inhibitor [3], serum antitryptase [4], antiplasmin [5], serum antiprotease [6]) is present in the plasma of all species which we have tested. It is not yet available in a pure state and therefore its chemical characteristics are unknown. This substance or substances in plasma inactivate fibrinolysin. It is not certain whether antifibrinolysin forms an inactive complex with fibrinolysin or whether the in-

1 Aided by a grant from the U. S. Public Health Service. activation is accomplished through alteration by antifibrinolysin of the fibrinolysin molecule.

Fibrinolysin (plasmin [7], serum tryptase [4], serum protease [6]) is produced in plasma by activation of a precursor $(s)$, profibrinolysin (plasminogen [7], tryptogen [4], lytic factor [8]). The nature of profibrinolysin is unknown. The proenzyme may exist as an inactive fibrinolysin molecule (7) or it may comprise a complex which includes fibrinolysin and antifibrinolysin (5). Activation conceivably occurs through the separation of antifibrinolysin from the fibrinolysin molecule.

Three methods of activation of profibrinolysin have been described. (a) Streptokinase (termed fibrinolysin by Garner and Tillett [9]) is derived from certain strains of streptococci (10). When incubated with plasma or crude thrombin and fibrinogen, streptokinase promotes the production of proteolytic activity but streptokinase alone does not appear to have proteolytic activity (7). (b) Treatment of plasma or serum with chloroform has long been known to result in the production of an active enzyme (11). (c) Recently Astrup and Permin $(2,12)$ have demonstrated that tissue washings from the cells of various organs from several animal species will activate profibrinolysin. They have called this activator "cytofibrinokinase." Permin has also reported that the stroma of human erythrocytes contains an activator of profibrinolysin (12).

\section{METHODS}

1. Preparation of special reagents. The systems are buffered at $\mathrm{pH} 7.25$ with imidazole. Ninety ml. of $0.1 \mathrm{~N} \mathrm{HCl}$ are used to dissolve $1.72 \mathrm{~g}$. of the salt. The $\mathrm{pH}$ is adjusted to 7.25 by means of $\mathrm{HCl}$ or $\mathrm{NaOH}$ and the solution made up to a 100 ml. volume with distilled water.

Thrombin Topical, Parke, Davis and Co., prepared by described methods (13-15), is employed 
in the clotting reactions. Two thousand Iowa units of dry thrombin are dissolved in a glycerolwater mixture containing equal parts by volume of water and glycerol. The glycerol is used for stabilization (16). This preparation is stable for several months when stored in a refrigerator at $5^{\circ} \mathrm{C}$.

For use in assaying fibrinolysin or antifibrinolysin the thrombin solution is poured to a depth of about $1 \mathrm{~cm}$. in a small test tube. Stirring rods, 3 to $4 \mathrm{~mm}$. in diameter, are immersed in this solution. Upon adding fibrinogen to the test samples a clot is formed by removing the stirring rod from the thrombin solution and rotating it through the solution containing the fibrinogen. This insures that the fibrinogen is uniformly distributed throughout the enzyme solution. The stirring rod is then removed from the clotting mixture. It requires about two to three seconds to add thrombin in this manner; a firm clot forms within 15 to 20 seconds.

2. Preparation and standardization of fibrinogen. For use in quantitative measurements fibrinogen of high purity and reproducible clottability is required. The bovine fibrinogen employed in these assays was prepared by the freezing-thawing technic which has been described in detail (17). Since the preparation is stable under proper storage conditions, a large quantity is prepared and stored in a series of test tubes, each tube containing a sufficient quantity for performing the assays required during one day. The physically separated fibrinogen is dissolved to a concentration of 0.5 to 1.0 per cent in 2 per cent $\mathrm{NaCl}$ containing 5 per cent of the imidazole buffer. A sodium chloride concentration greater than 0.9 per cent and the slightly alkaline $\mathrm{pH}$ assist in stabilization. The fibrinogen concentration is measured by the determination of tyrosine $(17,18)$. After dividing this stock solution and placing it into a series of pyrex test tubes it is quick frozen in an alcohol dry ice mixture in preparation for storage at $-20^{\circ} \mathrm{C}$.

Prior to each series of fibrinolysin or antifibrinolysin assays, the fibrinogen solution is thawed at $40^{\circ} \mathrm{C}$. without agitation and diluted to a fibrinogen concentration of 0.2 per cent in 0.9 per cent $\mathrm{NaCl}$. This solution is maintained at $40^{\circ} \mathrm{C}$. in a water bath except during pipetting procedures. If these precautions are followed, the fibrinogen remains stable and the clottability is not measurably altered during at least a 24-hour period.

The fibrin concentration in the standardized clot has been fixed in these studies at 0.1 per cent because this concentration produces a firm clot which collapses rapidly at the end-point. Variations in fibrinogen concentration in the standardized solution result in appreciable errors in the antifibrinolysin assay. In Table I are given the dissolving times of clots, containing sufficient fibrinolysin to cause a 0.1 per cent fibrin clot to dissolve in 120 seconds, when the fibrinogen concentration is varied between approximately 0.5 and 1 per cent. The curve when plotted on arithmetical graph paper is a straight line (19).

TABLE I

Lysis time of clots formed from varying concentrations of fibrinogen. All other factors are standardized as described in the text

The 0.1 per cent fibrinogen and 120 second lysis time are used as the standard for fibrinolysin assays.

\begin{tabular}{|c|}
\hline $\begin{array}{l}\text { Per cent of cor } \\
\text { fibrinoger }\end{array}$ \\
\hline $\begin{array}{l}0.046 \\
0.092 \\
0.139 \\
0.185 \\
0.231 \\
0.278 \\
0.370 \\
0.463 \\
0.648 \\
0.926\end{array}$ \\
\hline
\end{tabular}

Time required for clot to dissolve in seconds

3. Preparation and standardization of fibrinolysin solutions. The activity of the fibrinolysin supplied by E. C. Loomis and prepared by the method which has been described in detail (1) becomes stabilized in 15 to 20 minutes after it is dissolved in imidazole buffer and this activity remains relatively constant at room temperature for two to three hours thereafter. After three to four hours in solution at room temperature the activity gradually decreases. In view of these characteristics we permit about 50 minutes to elapse before attempting to standardize the fibrinolysin solutions and dissolve no more at one time than is required for a two to three hour series of assays.

For use in antifibrinolysin assays the unit activity of the fibrinolysin solution must be carefully standardized. We define one unit as the amount of fibrinolysin activity which will completely lyse 1 cc. of a 0.1 per cent fibrin clot at $28^{\circ}$ C. in 120 seconds in an isotonic saline solution buffered with 
TABLE II

Loss in activity of fibrinolysin solutions during 30 minute incubation at the indicated temperatures

$\begin{array}{cc}\begin{array}{c}\text { Temperature } \\ \text { degrees centigrade }\end{array} & \begin{array}{c}\text { Percentage loss } \\ \text { in activity }\end{array} \\ 45 & 90 \\ 40 & 85 \\ 35 & 75 \\ 30 & 17 \\ 28 & 0\end{array}$

imidazole. This unit requires three to five times as much fibrinolysin as the Loomis unit which is based upon the same dissolving time, but lyses a 0.3 per cent fibrin clot. Loomis, Ryder and George (1) carry out the lysis reaction at $45^{\circ} \mathrm{C}$. instead of $28^{\circ} \mathrm{C}$. Our reason for using the $28^{\circ} \mathrm{C}$. temperature is based upon fibrinolysin inactivation studies. As indicated in Table II a large percentage of the fibrinolysin activity is lost in 30 minutes at $45^{\circ} \mathrm{C}$. However, our tests have not included the shorter periods (two to five minutes) usually employed in the assay.

Figure 1 depicts the relationship between fibrinolysin activity and the breaking time of 0.1 per cent fibrin clots at $28^{\circ} \mathrm{C}$. The relationship is a straight line function when plotted on log paper. It is similar to the relationship between thrombin concentration and clotting time (20). Thus the

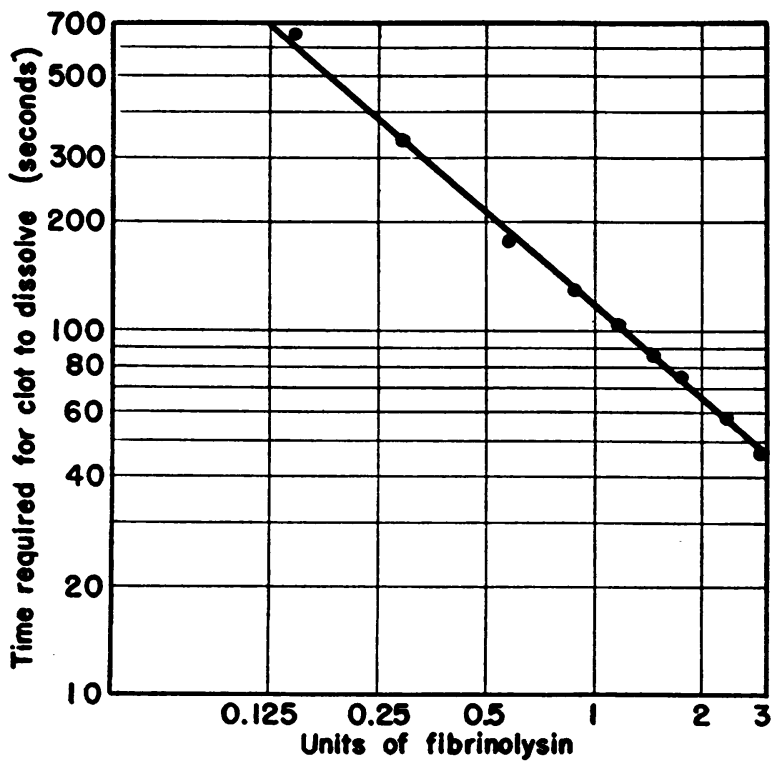

Fig. 1. Relationship between the Fibrinolysin Concentration and the Dissolving Time of a 0.1 Per Cent Fibrin Clot

All other variables are standardized as described in the text. logarithm of clotting time is inversely proportional to the logarithm of the thrombin concentration and the logarithm of the lysis time is inversely proportional to the logarithm of the fibrinolysin concentration.

In preparation for a fibrinolysin or an antifibrinolysin assay a measured volume of the imidazole buffer is added to a weighed quantity of the dry fibrinolysin. Solution is facilitated by stirring. Thirty minutes after adding the imidazole buffer to the fibrinolysin the material is centrifuged at 3,000 r.p.m. for 20 minutes. This procedure removes undissolved particles. The supernatant solution is used for assay.

To determine the unit activity of a fibrinolysin solution (Figure 1), $0.2 \mathrm{ml}$. is pipetted into a test tube, $50 \mathrm{~mm}$. $\times 8 \mathrm{~mm}$. I.D. As $0.2 \mathrm{ml}$. of 0.2 per cent fibrinogen is pipetted into the tube a stop watch is started. Thrombin is added by stirring rod as described above and the tube placed in a $28^{\circ} \mathrm{C}$. water bath. Thirty seconds after adding the fibrinogen a capillary tube, 0.5 to $1.0 \mathrm{~mm}$. I.D. with a beveled tip, is lowered gently down the side of the test tube. The capillary tube pushes the formed clot to one side and the beveled tip of the capillary tube rests on the bottom of the test tube. The stop watch is stopped at the end-point of the titration, when the liquid within the capillary tube passes the level of the surrounding liquid. The capillary tube end-point method was recently developed in this laboratory to eliminate errors which may occur from temperature changes and agitation when the method of tilting the tube is used to determine completion of lysis (19). The results which are given in this report, however, were obtained by means of the tilting method which gives essentially the same end-point but greater variation in duplicate and triplicate determinations.

The standarization of fibrinolysin for use in the antifibrinolysin assay is performed in the same manner except that the concentration of fibrinolysin in imidazole buffer is double that utilized in the fibrinolysin assay. One-tenth $\mathrm{ml}$. of this solution is added to the reaction tube followed by 0.1 $\mathrm{ml}$. of normal saline solution. Adjustment must be made in the fibrinolysin concentration to bring the activity, measured in this manner, to a lysis time of $120 \pm 5$ seconds. It is best to make the original fibrinolysin solution slightly more concentrated 
and add normal saline in adjusting to the required activity.

4. Collection and preparation of plasma. In each species seven parts of whole blood were mixed with 1 part of 1.85 per cent potassium oxalate. Hematocrit determinations were made and the blood was immediately centrifuged to remove the cells. The plasma was defibrinated by adding Thrombin Topical, Parke, Davis and Co., in the dry form and the fibrin removed with a glass stirring rod. If the antifibrinolysin assay could not be performed immediately, the plasma was stored at $-20^{\circ} \mathrm{C}$. until the following day.

All animals used for obtaining blood samples were in a state of good nutrition and exhibited no obvious pathology. When anesthesia, ether or nembutal, was employed, as in the cat, dog and opossum, blood was taken immediately after the induction of anesthesia. Macfarlane and Biggs (21) have indicated that fibrinolytic activity occurs as frequently in the plasma of human patients during anxiety states as during anesthesia. We have not yet investigated the effect of anesthesia on the antifibrinolysin activity of plasma.

The antifibrinolysin assay curves are based upon the pooled blood from several individuals of each species. The number of individuals making up the pooled samples are as follows: alligator 3 , guinea pig 6 , cow 4 , rat 10 , frog 24 , human 10 , cat 4 , dog 9 , rabbit 12, opossum 3 , chicken 6 and pigeon 6 . In addition, determinations of antifibrinolysin in the plasma of individuals of several species (bovine, man, dog, chicken) indicated that the antifibrinolysin activity in the plasma of a normal individual closely approximates the value obtained from an analysis of the pooled plasma of that species.

5. Assay of plasma antifibrinolysin. Fibrinolysin is relatively stable in solution at $\mathrm{pH} 7.2$ over a period of two to three hours if the temperature of the solution remains at $28^{\circ} \mathrm{C}$. or below. However, if diluted plasma is added to the fibrinolysin solution, a portion of the fibrinolysin activity disappears rapidly. If the added plasma is sufficiently dilute, equilibrium is reached within five to 10 minutes and thereafter either no additional fibrinolysin activity disappears from the system or the decrease in activity is relatively slow. In Figure 2 is plotted time against lysis time for a series of diluted human plasmas. The time curves for chicken plasma are included in another report
(19). Although actual tests have not been made, all available evidence indicates that the antifibrinolysin in the plasmas of other animals acts in a similar manner.

The measurement of the amount of fibrinolysin remaining after 60 minutes in the presence of antifibrinolysin determines the concentration activity of the antifibrinolysin. More concentrated plasma (higher antifibrinolysin concentration) inactivates a large percentage of the fibrinolysin present while dilute plasma (lower antifibrinolysin concentration) inactivates less fibrinolysin. Therefore if fibrinolysin of standardized activity is allowed to react with antifibrinolysin until equilibrium occurs, a long dissolving time of a standard clot indicates a large amount of antifibrinolysin while a short dissolving time indicates a low antifibrinolysin activity. The assay of antifibrinolysin is based upon these considerations.

Five units of plasma antifibrinolysin are defined as the quantity of antifibrinolysin which within 60 minutes at $28^{\circ} \mathrm{C}$. and at $\mathrm{pH} 7.2$ will inactivate 50 per cent of the fibrinolysin in $1 \mathrm{ml}$. of a standard solution containing 1 unit of fibrinolysin per $\mathrm{ml}$.

To $0.1 \mathrm{ml}$. of the double strength standardized fibrinolysin solution in a $50 \times 8 \mathrm{~mm}$. test tube is added $0.1 \mathrm{ml}$. of diluted plasma. The tube is then

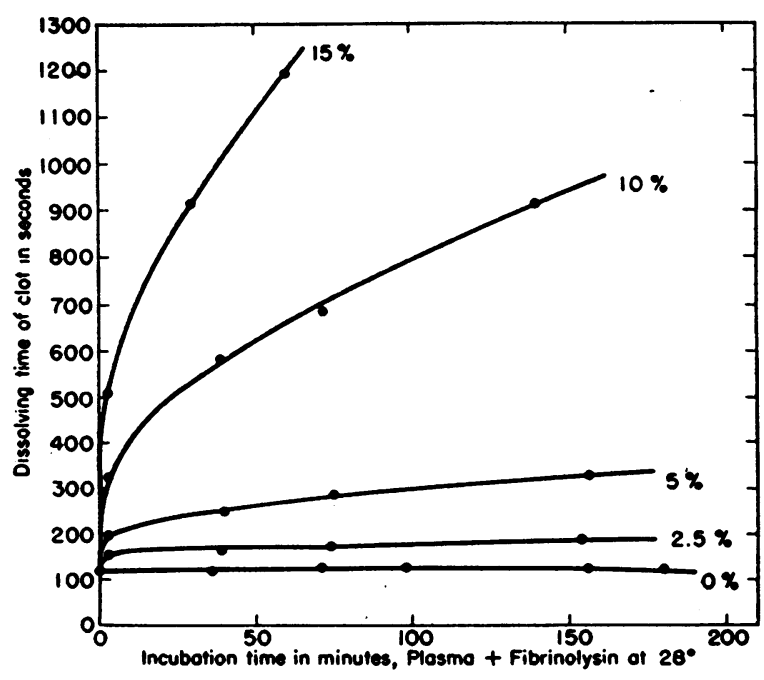

Fig. 2. The Dissolving Time of Standardized Clots Containing Human Plasma Concentrations as Indicated on Each Curve

The incubation time of fibrinolysin with plasma is measured on the abscissa. 
placed in a water bath at $28^{\circ} \mathrm{C}$. for $60 \mathrm{~min}$ utes. After 60 minutes' incubation the tube is removed from the water bath and $0.2 \mathrm{ml}$. of the standard 0.2 per cent fibrinogen solution added as the stop watch is started. Thrombin is added by stirring rod as above and the solution mixed. The tube is then returned to the water bath. The end-point is the complete dissolution of the clot (see No. 3 above). The elapsed time between the addition of fibrinogen and the dissoluton of the clot is recorded. All determinations are carried out in triplicate and are based on the average for the three measurements.

6. Assay curves. The curves in Figures 2 and 3 for the plasmas of the various animal species studied are based upon the plot of a series of plasma dilutions against the dissolving time of the standard clot. In each curve the coordinate
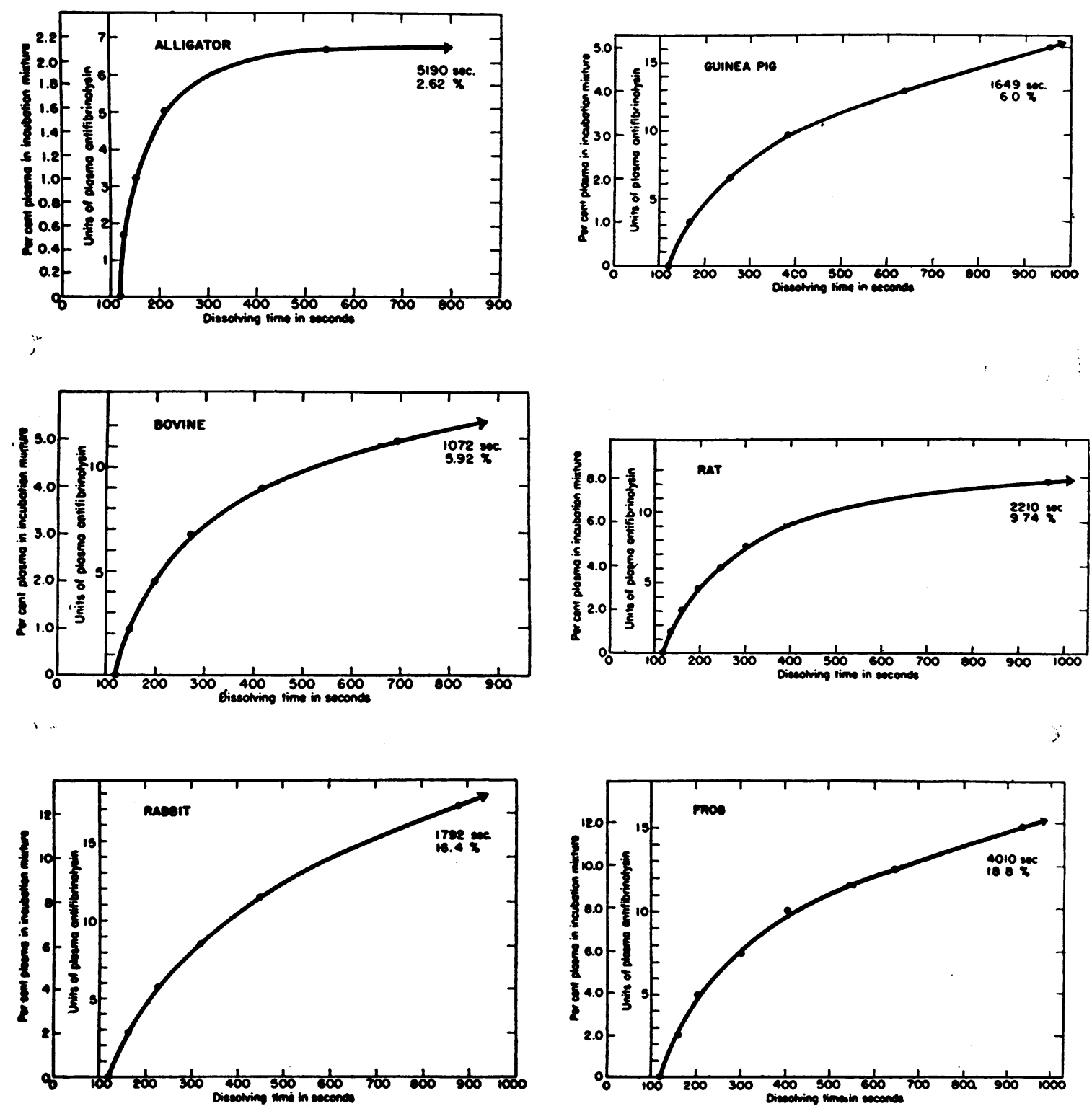

Fig. 3. The Dissolving Time of Standardized Clots Containing Varying Percentages of the Plasmas of Each Species Studied

See text for details and interpretation. 
for the 210 second dissolving time has been determined accurately. The 210 second dissolving time corresponds to one-half unit of fibrinolysin activity in Figure 1. The coordinate has therefore been extended from the 210 second dissolving time on the antifibrinolysin concentration curves to the ordinate and this value designated as 5 units of antifibrinolysin activity. The ordinate has been further divided into fractions and multiples of $\mathbf{5}$ units.
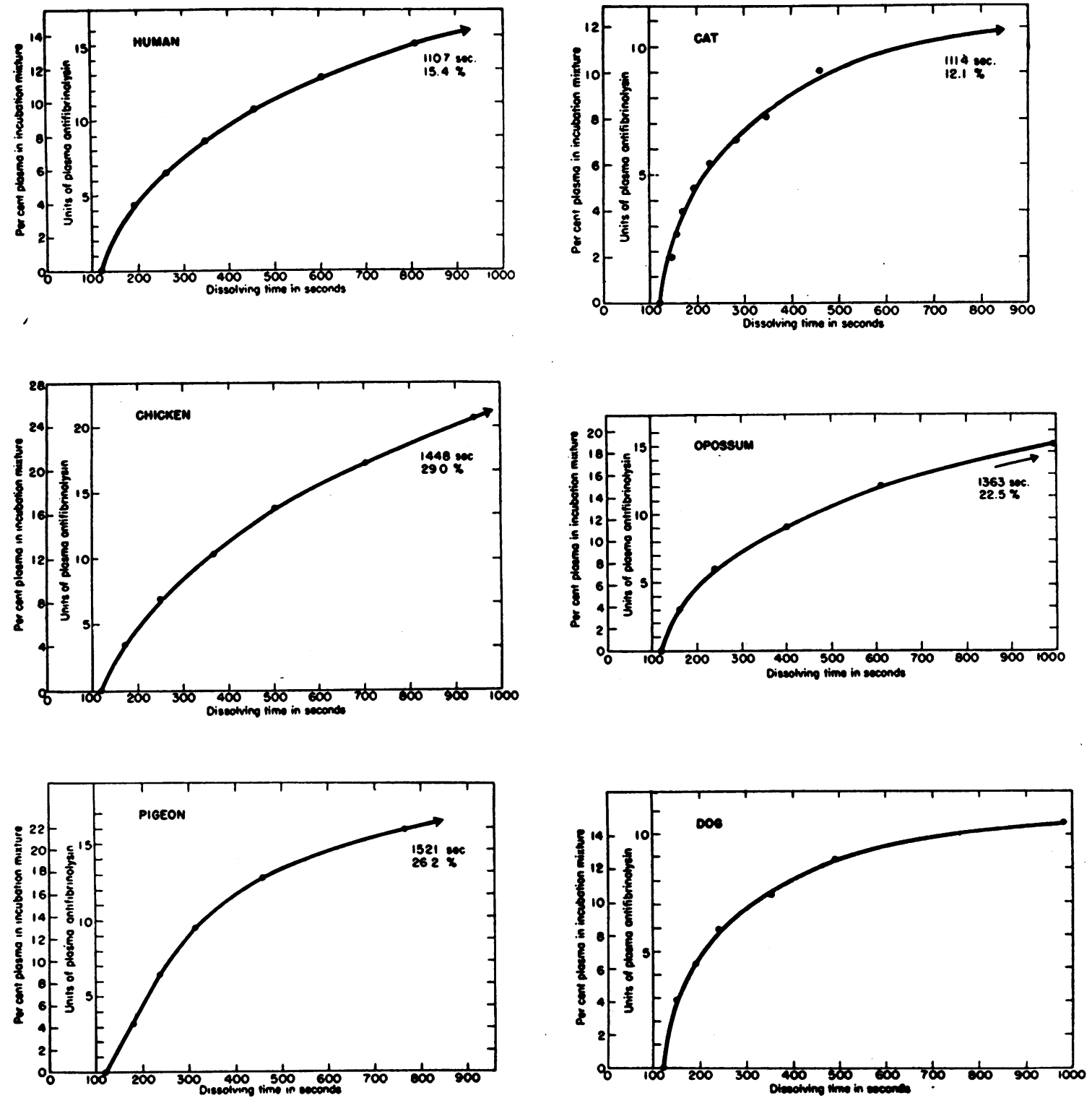

Fig. 4. The Dissolving Time of Standardized Clots Containing Varying Percentages of the Plasmas of Each Species Studied

See text for details and interpretation.

To assay the antifibrinolysin activity in the plasma of any of the species which we have studied, the plasma is obtained and prepared as described under No. 4 above. Dilutions of the plasma are made such that the dissolving time of the standard clot is less than 300 seconds. The plasma and the standardized fibrinolysin are incubated at $28^{\circ}$ C. for 60 minutes. Fibrinogen and thrombin are added and the dissolving time of the clot determined at $28^{\circ} \mathrm{C}$. By reference to the curve for
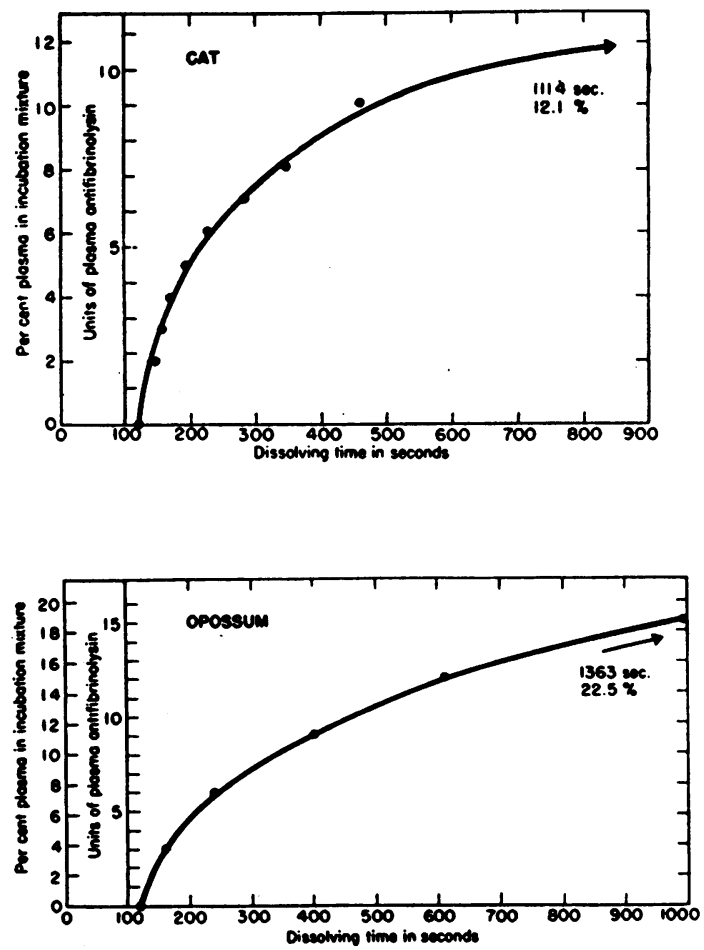
the species studied, the unit concentration of plasma antifibrinolysin may be read off on the ordinate when the dissolving time is known. The number of units determined from the curve is multiplied by the dilution factor to give the antifibrinolysin unit concentration in $1 \mathrm{ml}$. of undiluted plasma. The dilution factor includes dilution of the plasma to bring the dissolving time of the clot into the less than 300 second range and the dilution resulting from the addition of oxalate at the time the blood was drawn. For example, if the volume of the plasma-oxalate mixture in the incubation mixture is one-tenth of the total volume, seven parts of blood were added to one part of oxalate at the time of bleeding and the hematocrit (not corrected for oxalate) was 40 , then the dilution is $\frac{10}{1} \times \frac{(1.00-0.40) 8}{((1.00-0.40) 8)-1}=12.63$. If 6 units have been found in this sample by titration, then there are $6 \times 12.63=76$ units of antifibrinolysin per ml. of undiluted plasma.

The relationships between antifibrinolysin activity units and plasma concentration for the different species studied, depicted in Figures 3 and 4, have been arranged in the order of decreasing antifibrinolysin activity. Thus when bovine fibrinolysin is the substrate, alligator plasma is found to have the greatest antifibrinolysin unit activity per $\mathrm{ml}$. of plasma and in decreasing order of activity the species are: guinea pig, bovine, rat, rabbit, frog, human, cat, chicken, opossum, pigeon, and dog. This order is based upon the plasma concentration at an antifibrinolysin activity level of 5 units. Slight variations in the species order would result from using higher or lower unit activities as the reference point. This discrepancy is the result of slight differences in the character of the curves from species to species.

\section{DISCUSSION}

The methods presented in this report make possible a comparative study of the antifibrinolysin activity in different animals and should prove to be useful tools in the determination of physiological and pathological factors which alter the concentration of this plasma component. In addition, experimental alteration of the plasma antifibrinolysin activity level may be followed through use of this assay method and the physiological significance of such changes in antifibrinolysin activity determined by observation of the effects upon a single tissue or upon the economy of the entire organism.

At the present time it is not practical to assay for the fibrinolytic activity of plasma. Methods which have been suggested $(5,22)$ require excessively long periods of time and indicate little more than the presence or absence of fibrinolytic activity in an in vitro system. Evidence obtained through the use of such methods indicates that free fibrinolytic activity may occur in plasma $(5,22-25)$. However, since an excess of antifibrinolysin in the plasma rapidly inactivates free fibrinolysin, an assay indicating no antifibrinolysin activity would be an indication that free fibrinolysin could exist. Our assays which include studies of the antifibrinolysin activity levels in several hundred animals of different species and under various experimental conditions as well as the plasma levels in normal and pathological human individuals have failed in all cases to indicate an absence of antifibrinolysin. It therefore appears that antifibrinolysin is commonly in excess. From available evidence it is not possible to ascertain whether plasmas which are fibrinolytically active result from an increase in fibrinolysin concentration or from a decrease in the antifibrinolysin activity.

The physiological significance of differences between species in plasma antifibrinolysin activity and in the character of the curves presented in Figures 3 and 4 cannot be determined from the available data. In the assay presented here, the factors existing in the plasma of different species are permitted partially to inactivate bovine fibrinolysin. The fibrinolysin molecule may differ in each species or it may be identical. In the same way antifibrinolysin may differ from species to species, or the same molecule may be present in the plasma of all species studied. It is possible that the relative antifibrinolysin activity in the plasmas of the different species would fall in a different order if the fibrinolysin were derived from the plasma of some species not belonging to the bovine genus. However, even though the fibrinolysin molecule may differ from species to species, the fact that the plasma of all species studied will inactivate bovine fibrinolysin makes possible comparative studies of antifibrinolysin activity in any one of the several species investigated. 


\section{SUM MARY}

1. Methods are described which make possible the study of antifibrinolysin activity variations in the plasma of the alligator, guinea pig, cow, frog, rat, rabbit, man, cat, dog, opossum, pigeon, and chicken. The antifibrinolysin in the plasma of each species inactivates bovine fibrinolysin.

2. Fundamental relationships, important in the assay, are described. These include (1) the relationship between the fibrinogen concentration and the dissolving time of clots, (2) the inverse relationship between the logarithm of the fibrinolysin concentration and the logarithm of the dissolving time of the clots, (3) the equilibrium reaction between fibrinolysin and diluted plasma antifibrinolysin and (4) the character of the curves for the different species when the plasma concentration is plotted against the dissolving time of the clots.

3. Using bovine fibrinolysin as the enzyme the sequence of decreasing plasma antifibrinolysin activity by species is alligator, guinea pig, bovine, rat, rabbit, frog, human, cat, chicken, opossum, pigeon, and dog.

We wish to thank Eugene C. Loomis for generous supplies of fibrinolysin and Parke, Davis and Company for financial support of research in Physiology.

\section{BIBLIOGRAPHY}

1. Loomis, E. C., George, C., Jr., and Ryder, A., Fibrinolysin: Nomenclature, unit, assay, preparation and properties. Arch. Biochem., 1947, 12, 1.

2. Astrup, T., and Permin, P. M., Fibrinolysis in the animal organism. Nature, 1947, 159, 681.

3. Guest, M. M., Daly, B. M., Ware, A. G., and Seegers, W. H., Quantitative measurements of a fibrinolysin inhibitor in the plasma of various species. Fed. Proc., 1947, 6, 118.

4. Ferguson, J. H., Nomenclature of perenteral proteases. Science, 1947, 105, 488.

5. Macfarlane, R. G., and Pilling, J., Observations on fibrinolysis; plasminogen, plasmin, and antiplasmin content of human blood. Lancet, 1946, 2, 562.

6. Kaplan, M. H., Studies of streptococcal fibrinolysis. II. The inhibition of streptococcal fibrinolysis by antifibrinolysin and antiprotease. J. Clin. Invest., 1946, 25, 337.

7. Christiansen, L. R., and Macleod, C. M., A proteolytic enzyme of serum: Characterization, activation and reaction with inhibitors. J. Gen. Physiol., 1945, 28, 559.

8. Milstone, H., A factor in normal human blood which participates in streptococcal fibrinolysis. J. Immunol., 1941, 42, 109.

9. Garner, R. L., and Tillett, W. S., Biochemical studies on the fibrinolytic activity of hemolytic streptococci. J. Exper. Med., 1934, 60, 239.

10. Tillett, W. S., The fibrinolytic activity of hemolytic streptococci. Bact. Rev., 1938, 2, 161.

11. Delezenne, C., and Pozerski, E., Action proteolytique du sérum sanguin prealablement traité par le chloroforme. Compt. rend. Soc. de biol., 1903, 55, 690.

12. Permin, P. M., Properties of the fibrinokinase-fibrinolysin system. Nature, 1947, 160, 571.

13. Seegers, W. H., Purification of prothrombin and thrombin: Chemical properties of purified preparations. J. Biol. Chem., 1940, 136, 103.

14. Seegers, W. H., and McGinty, D. A., Further purification of thrombin; probable purity of products. J. Biol. Chem., 1942, 146, 511.

15. Loomis, E. C., and Seegers, W. H., Purified prothrombin: Factors which influence its activation. Arch. Biochem., 1944, 5, 265.

16. Seegers, W. H., Purified prothrombin and thrombin: Stabilization of aqueous solutions. Arch. Biochem., 1944, 3, 363.

17. Ware, A. G., Guest, M. M., and Seegers, W. H., Fibrinogen: With special reference to its preparation and certain properties of the product. Arch. Biochem., 1947, 13, 231.

18. Folin, O., and Ciocalteu, V., On tyrosine and tryptophane determinations in proteins. J. Biol. Chem., 1927, 73, 627.

19. Guest, M. M., Ware, A. G., and Seegers, W. H., A quantitative study of antifibrinolysin in chick plasma: Increase in antifibrinolysin activity during pteroylglutamic acid deficiency. Am. J. Physiol., 1947, 150, 661.

20. Seegers, W. H., and Smith, H. P., Factors which influence the activity of purified thrombin. Am. J. Physiol., 1942, 137, 348.

21. Macfarlane, R. G., and Biggs, R., Observations on fibrinolysis. Lancet, 1946, 2, 862.

22. Tagnon, H. J., Levenson, S. M., Davidson, C. S., and Taylor, F. H. L., The occurrence of fibrinolysis in shock, with observations on the prothrombin time and the plasma fibrinogen during hemorrhagic shock. Am. J. Med. Sci., 1946, 211, 88.

23. Judine, S. S., La transfusion du sang de cadavre aux êtres humains. Presse méd., 1936, 44, 68.

24. Macfarlane, R. G., Fibrinolysis following operation. Lancet, 1937, 1, 10.

25. Biggs, R., Macfarlane, R. G., and Pilling, J., Observations on fibrinolysis; experimental activity produced by exercise or adrenaline. Lancet, 1947, 1, 402. 